\title{
A novel ecological role of the Firmicutes identified in thermophilic microbial fuel cells
}

\author{
Kelly C Wrighton ${ }^{1}$, Peter Agbo ${ }^{1}$, Falk Warnecke ${ }^{2}$, Karrie A Weber ${ }^{1}$, Eoin L Brodie ${ }^{3}$, \\ Todd Z DeSantis ${ }^{3}$, Philip Hugenholtz ${ }^{2}$, Gary L Andersen ${ }^{3}$ and John D Coates ${ }^{1,3}$ \\ ${ }^{1}$ Department of Plant and Microbial Biology, University of California, Berkeley CA, USA; ${ }^{2}$ Microbial Ecology \\ Program, DOE Joint Genome Institute, Walnut Creek, CA, USA and ${ }^{3}$ Earth Sciences Division, Ecology \\ Department, Lawrence Berkeley National Laboratory, Berkeley, CA, USA
}

\begin{abstract}
Significant effort is currently focused on microbial fuel cells (MFCs) as a source of renewable energy. Most studies concentrate on operation at mesophilic temperatures. However, anaerobic digestion studies have reported on the superiority of thermophilic operation and demonstrated a net energy gain in terms of methane yield. As such, our studies focused on MFC operation and microbiology at $55^{\circ} \mathrm{C}$. Over a 100-day operation, these MFCs were stable and achieved a power density of $37 \mathrm{~mW} \mathrm{~m}^{-2}$ with a coulombic efficiency of $89 \%$. To infer activity and taxonomic identity of dominant members of the electricity-producing community, we performed phylogenetic microarray and clone library analysis with small subunit ribosomal RNA (16S rRNA) and ribosomal RNA gene (16S rDNA). The results illustrated the dominance ( $80 \%$ of clone library sequences) of the Firmicutes in electricity production. Similarly, rRNA sequences from Firmicutes accounted for $50 \%$ of those taxa that increased in relative abundance from current-producing MFCs, implying their functional role in current production. We complemented these analyses by isolating the first organisms from a thermophilic MFC. One of the isolates, a Firmicutes Thermincola sp. strain JR, not only produced more current than known organisms $(0.42 \mathrm{~mA})$ in an $\mathrm{H}$-cell system but also represented the first demonstration of direct anode reduction by a member of this phylum. Our research illustrates the importance of using a variety of molecular and culture-based methods to reliably characterize bacterial communities. Consequently, we revealed a previously unidentified functional role for Gram-positive bacteria in MFC current generation.

The ISME Journal (2008) 2, 1146-1156; doi:10.1038/ismej.2008.48; published online 4 September 2008

Subject Category: microbial ecology and functional diversity of natural habitats

Keywords: biofuel; biofilm; extracellular electron transfer; iron reduction; anode
\end{abstract}

\section{Introduction}

Microorganisms in a microbial fuel cell (MFC) catalyze the conversion of organic matter into electrical energy. As carbon neutral technology, MFCs represent a novel method for renewable energy production and wastewater treatment mitigating greenhouse gas emissions and producing electricity directly from biomass. Although research endeavors are committed to MFC optimization, these are largely focused on increasing power densities through system design. In contrast, less attention has focused on the microbiology driving electricity production. Given that energy generation

Correspondence: JD Coates, Department of Plant and Microbial Biology, University of California, Berkeley, 271 Koshland Hall, Berkeley, CA 94720, USA.

E-mail: jcoates@nature.berkeley.edu

Received 6 March 2008; revised 24 April 2008; accepted 24 April 2008; published online 4 September 2008 is governed by biological activity, understanding the relevant microbiology is crucial to increasing the power yield and applicability of these systems (Lovley, 2006).

To date, knowledge of the microbial physiology of electricity production is hindered by the limited number of current-producing isolates in pure culture. With the exception of Geothrix fermentans, an Acidobacteria, studied isolates capable of direct electricity production are confined to the Proteobacteria (Kim et al., 1999, 2002; Bond and Lovley, 2003; Chaudhuri and Lovley, 2003; Holmes et al., 2004; Rabaey et al., 2005). Furthermore, despite the broad phylogenetic diversity of bacteria known to transfer electrons onto solid phase electron acceptors (Weber et al., 2006), studies exploring electron transfer in MFCs predominately use the Fe(III)-reducing Geobacter and Shewanella species, members of the delta and gamma Proteobacteria, respectively (Kim et al., 1999; Bond and Lovley, 
2003; Reguera et al., 2005; Gorby et al., 2006; Bretschger et al., 2007). This discrepancy between culture-independent and culture methods suggests that the true diversity of electricity-producing organisms remains to be discovered. This is further supported by culture-independent studies, which indicate that the phylogenetic diversity of MFC microbial communities far exceeds that of electrochemically active isolates. Unfortunately, these community studies (Bond et al., 2002; Lee et al., 2003; Holmes et al., 2004; Kim et al., 2004; Rabaey et al., 2004; Aelterman et al., 2006; Jong et al., 2006; Kim et al., 2006) were hampered by the use of lowresolution DNA fingerprinting techniques targeting the $16 \mathrm{~S}$ ribosomal RNA gene (16S rDNA), the presence of which does not necessarily demonstrate electrochemical activity but may simply be an artifact of gene persistence in the environment (Manefield et al., 2002). Furthermore, because these studies focused on gene presence (16S rDNA) rather than gene expression (16S rRNA) they could not elucidate active members of the anode biofilm. However, in spite of these limitations, the broader microbial diversity identified in MFCs by molecular approaches highlights our limited understanding of the species involved in power generation and emphasizes the need for an in-depth characterization of the functional microbiology of anode communities.

In this study, we use several complementary approaches to assess microbial diversity and function of anode biofilm communities. To expand the known phylogenetic diversity of electricityproducing organisms, we concentrated on MFCs operated at $55{ }^{\circ} \mathrm{C}$, under which conditions neither Geobacter nor Shewanella species can survive (Lovley, 2000). Moreover, as Proteobacteria represent a minor constituent of thermophilic anaerobic digester microbiota (Sekiguchi et al., 1998; Jong et al., 2006), MFCs inoculated with thermophilic digester sludge offer a high likelihood of isolating novel electricity-producing bacteria. Additional benefits also exist for operating fuel cells at elevated temperatures including potentially higher energy yields, easier maintenance of anaerobic reducing conditions because of the lower solubility of $\mathrm{O}_{2}$ at elevated temperatures and thermal removal of most known pathogens (Jong et al., 2006).

To define the microbial ecology of an undefined system, we utilized several approaches to minimize the individual bias of any one method. We used clone library sequencing and a novel high-density oligonucleotide microarray (PhyloChip) (Brodie et al., 2006, 2007; DeSantis et al., 2007) to identify organisms enriched only in current-producing reactors. As the first application of the PhyloChip to anode biofilm communities, we provide a higher resolution analysis of microbial community composition uncovering a far greater diversity than observed previously. Additionally, although previous MFC community analyses have included a temporal context, in contrast to results presented here, they did not account for bacterial populations that were also enriched on non-current producing electrodes. Also, we distinguished active from persistent members of the electricity-producing community by monitoring 16S rRNA expression in addition to cataloging 16S rRNA gene presence (Duineveld et al., 2001; Manefield et al., 2002; Lueders et al., 2004). Finally, to connect metabolic function and taxonomic identity, we isolated dominant members of the current generating bacterial community and demonstrated their ability to produce electricity. By understanding the potential function of these organisms in pure culture, we infer their function within the electrode community.

\section{Materials and methods}

\section{MFC construction}

In an MFC bacteria act as catalysts: converting organic matter into electrical energy. MFCs are composed of two physically distinct regions: the anode, where bacteria donate electrons and the cathode, where electrons are transferred to a final electron acceptor. Bacteria liberate electrons from oxidation of organic compounds onto the anode where they flow through an external circuit, which contains an external resistor, to the cathode. Bacterial-mediated electron flow, or current, is governed by the electrochemical potential between the final bacterial respiratory enzyme and the cathode electron acceptor. To complete the internal circuit, protons diffuse from the anode to the cathode through a proton permeable membrane. At the cathode, the electrons and protons combine to reduce the terminal electron acceptor, which in many applications is oxygen.

The MFCs were designed and constructed as described previously (Thrash et al., 2007). The anodic chamber was filled with $250 \mathrm{ml}$ of presterilized, anoxic $30 \mathrm{~mm}$ bicarbonate buffer ( $\mathrm{pH}$ 6.8) and for certain reactors amended with $10 \mathrm{mM}$ acetate as the sole electron donor. In all cases, the cathodic chamber was filled with $250 \mathrm{ml}$ of $30 \mathrm{mM}$ TRIS buffer ( $\mathrm{pH}$ 6.8). The anode chamber was bubbled continuously with filtered $(0.22 \mu \mathrm{m}$ pore size $)$ $\mathrm{N}_{2}: \mathrm{CO}_{2}$ (80:20; vol:vol) gas to ensure anaerobic operation and maintenance of $\mathrm{pH}$ throughout operation, although the cathode chamber was bubbled continuously with air. MFCs were maintained at $56{ }^{\circ} \mathrm{C}$ over the course of the experiments using heating coils inserted into the anode chamber.

\section{MFC inoculum}

The inoculum was collected from an operational thermophilic methanogenic anaerobic digester. In the laboratory, anaerobic digester sludge was centrifuged $(10000 \mathrm{~g} ; 15 \mathrm{~min})$ and washed with bicarbonate buffer (30 mM, pH 6.8) under an $\mathrm{N}_{2}: \mathrm{CO}_{2}$ (80:20; vol:vol) headspace to remove soluble 
electron acceptors and electrochemically active compounds. The pellet was suspended in bicarbonate freshwater media lacking electron donor and acceptor to create a cell slurry under a headspace of $\mathrm{N}_{2}: \mathrm{CO}_{2}$ (80:20; vol:vol) (Thrash et al., 2007) and incubated at $56^{\circ} \mathrm{C}$. The removal of endogenous electron donors was monitored by cessation of biogas production as well as a depletion of organic acids. Upon the removal of endogenous electron donors, four MFCs were inoculated (10:90; vol:vol) with the laboratory acclimated anaerobic digester sludge.

To evaluate the electrochemical activity of isolates Geobacillus sp. strain S2E and Thermincola sp. strain JR, pure cultures were washed and inoculated into the MFC. Cells were grown anaerobically at $56{ }^{\circ} \mathrm{C}$ in $1 \mathrm{l}$ vol using acetate $(10 \mathrm{mM})$ as the electron donor and AQDS (10 mM) as the electron acceptor using standard anaerobic techniques and basal bicarbonate media. Bacterial cells were harvested upon the reduction of AQDS by centrifugation under an anoxic atmosphere (80:20; $\mathrm{N}_{2}: \mathrm{CO}_{2}$ atmosphere).

\section{MFC experimental design}

Acetate was chosen as the electron donor since it is not readily fermentable, it is the dominant electron donor in anaerobic systems, and our primary goal was to identify novel bacteria capable of anodic respiration (Lovley, 2006). The experimental design included three treatments: $10 \mathrm{mM}$ acetate amendment with circuit (two reactors), non-amended with circuit control (two reactors) and $10 \mathrm{mM}$ acetate amended without circuit control (one reactor). The non-amended reactors control for redox active components in sludge slurry, confirmed the absence of electron donors in the sludge inoculum and identify bacterial populations residing on the anode independent of acetate-dependent current production. The open circuit control normalizes for microbial populations that oxidize acetate independent of electron transfer to the anode.

\section{Electrochemical characterization}

Voltage ( $\mathrm{E}_{\text {cell }}$ or cell potential) was measured across a fixed external resistor $\left(\mathrm{R}_{\text {ext }}\right)$ and logged using Chart 4.0 software (ADInstruments, CA, USA). Data were recorded every $10 \mathrm{~min}$ for 100 days. Current $(I)$ was calculated from measured voltages using Ohm's law $\left(I=\mathrm{E}_{\text {cell }} / \mathrm{R}_{\text {ext }}\right)$. With the exception of polarization curve (supporting information), external resistance was kept constant at $470 \Omega$. Maximum overall power $(P)$ was calculated as $P=\mathrm{E}_{\text {cell }}^{2} \mathrm{Max} / \mathrm{R}_{\text {ext }}$. Coulombic efficiency, or electrons in acetate that are recovered as current, was calculated in duplicate and the mean value reported. The actual coulombs transferred was determined by integrating current $(\mathrm{A}=\mathrm{C} / \mathrm{S})$ vs time (S) plots. The total amount of electrons available was calculated by [(concentration of added acetate, $\mathrm{mM}) \times(1 \mathrm{~mol}$ Ac/1000 $\mathrm{mMol}$ Ac $) \times$ $(8 \mathrm{~mol} \mathrm{e}-/ 1 \mathrm{~mol} \mathrm{Ac}) \times(96485 \mathrm{C} / \mathrm{mol} \mathrm{e}-)]$.

\section{Nucleic acid isolation}

To characterize changes in the microbial community after 100 days of current production, anode electrodes were removed from the four reactors. The surface of the anode was rinsed to remove debris using sterile nuclease-free water. The graphite electrode was scraped into sterile DNA/RNA-free $2 \mathrm{ml}$ centrifuge tubes with a sterile razor blade. Nucleic acids were extracted from the graphite using a modified CTAB extraction buffer (equal volumes of $10 \% \mathrm{CTAB}$ in $0.7 \mathrm{M} \mathrm{NaCl}$ and $240 \mathrm{~mm}$ potassium phosphate buffer, $\mathrm{pH}$ 8). $0.3 \mathrm{~g}$ of liquid nitrogen frozen graphite was added to Lysing Matrix E tubes (Bio101 Systems, CA, USA) containing $0.5 \mathrm{ml}$ CTAB buffer, $0.1 \mathrm{mg} \mathrm{ml}^{-1}$ proteinase $\mathrm{K}$ (Ambion, TX, USA). Samples were mechanically lysed by beadbeating for $20 \mathrm{~s}$ at 550 r.p.m. Nucleic acids were extracted once with phenol:chloroform:isoamyl alcohol (25:24:1) followed by a second extraction with chloroform:isoamyl alcohol (24:1). Nucleic acids were precipitated overnight with isopropanol at -20 degrees and pellets rinsed with $70 \%$ ethanol. Five extractions were performed for each anode sample; pellets from each sample were combined and resuspended in $100 \mu \mathrm{l}$ TE buffer. RNA and DNA were purified using the All Prep DNA/RNA kit (Qiagen, CA, USA). Isolated RNA was purified from any potentially contaminating DNA using on-column DNAse digestion with DNAse-free RNAse set (Qiagen). To confirm the purity of RNA and lack of DNA contamination, PCR amplifications were performed using non-reverse transcribed DNAse-treated RNA as a control. Only samples demonstrating negative results, no amplification, were reverse transcribed into cDNA using Superscript II reverse transcriptase per the manufacturers protocol (Invitrogen, CA, USA).

16S rRNA gene amplification for PhyloChip and isolate identification

The 16S rRNA gene was amplified from gDNA extracts using universal primers 27F (5'-AGAGTTT GATCCTGGCTCAG) and 1492R (5'-GGTTACCTTGT TACGACTT) for bacteria and 4Fa (5'-TCCGGT TGATCCTGCCRG-3') combined with 1492R for archaea. PCR amplifications were set up according to protocol outlined previously (Flanagan et al., 2007). PCR products for each sample were combined, concentrated by precipitation and resuspended in sterile nuclease-free water.

\section{$16 S$ rRNA amplicon analysis by PhyloChip hybridization}

The pooled PCR product was spiked with known concentrations of synthetic 16S rRNA gene fragments and non-16S rRNA gene fragments as internal standards for normalization, with quantities ranging from $5.02 \times 10^{8}$ to $7.29 \times 10^{10}$ molecules applied to the final hybridization mix. Target 
fragmentation, biotin labeling, PhyloChip hybridization, scanning and staining, as well as background subtraction, noise calculation and detection and quantification criteria were performed as reported (Flanagan et al., 2007). A taxon was considered present in the sample when $90 \%$ or more of its assigned probe pairs for its corresponding probe set were positive (positive fraction $\geqslant 0.90$ ).

\section{Identifying anode community membership and dynamic populations by PhyloChip}

A total of 10 PhyloChip arrays were performed including DNA arrays from time zero inoculum (three), final DNA arrays from each of the four reactors (two experimental, one open circuit control and one no acetate control) and final cDNA arrays (two experimental and one no acetate control). Three randomly chosen replicate samples from time zero were analyzed with PhyloChip microarrays to confirm that reactors were inoculated with bacterial communities containing a similar composition; verifying bacterial heterogeneity was not a factor contributing to differences in the anode communities.

Bacterial communities were present on anodes of current-producing reactors and reactors that failed to produce current. Cluster analyses were performed on PhyloChip outputs from RNA and DNA arrays. Sample clustering was also confirmed by the $\mathrm{R}$ statistical programming environment using the function 'heatmap' within the package 'made4'. Both samples and OTUs were clustered with one Pearson's correlation as the distance metric and UPGMA as the linkage method (Brodie et al., 2007). Given the high similarity with replicate reactors in the current-producing reactors and time zero treatments, overall hybridization intensity values for these treatments represent the average of the HybScores from the reactors within the treatment group.

\section{Identifying dominant anode members by clone library sequencing}

Clone libraries were generated for each treatment (time zero inoculum, experimental, open circuit control, no acetate control) from pooled DNA PCR products. Bacterial $16 \mathrm{~S}$ rRNA amplicon pools amplified with universal primers 27F (5'-AGAGTTT GATCCTGGCTCAG) and 1392R (5'-ACGGGCGGTG TGTAC) were ligated into pCR4-TOPO vectors (Invitrogen, CA, USA). Ligated plasmids were transformed into Escherichia coli TOP10 chemically competent cells according to the manufacturer's recommended protocol (Invitrogen, CA, USA). Clones were randomly selected by a robotic picker and inserts were sequenced bidirectionally using M13 vector specific primers. Sequences were primer and vector screened using cross_match, quality scored using Phred and assembled into contigs using Phrap. Sequences were trimmed to retain only bases
Phred $\geqslant$ q20 and high quality contigs were tested for chimeras (five of which were removed from further analysis) using Bellerophon version 3 (DeSantis et al., 2006). To generate libraries for each sample, the respective full-length 16S RNA PCR products were cloned into pCR4-TOPO vectors (Invitrogen) according to the manufacturer's instructions. A total of 192 transformants from each library were picked randomly. Double-ended sequencing reactions of the entire 16S RNA sequence were carried out with PE BigDye terminator chemistry (Perkin Elmer) and resolved with an ABI PRISM 3730 capillary DNA sequencer (Applied Biosystems) integrated into a pipeline (E Kirton, unpublished).

\section{Bacterial isolation}

All enrichments and isolates were incubated at $56{ }^{\circ} \mathrm{C}$. To obtain bacteria adapted to utilizing insoluble electron acceptors, electrode scrapings were inoculated into fresh water media (Coates et al., 1996) amended with poorly crystalline Fe(III) oxide (Lovley and Philips, 1986) as the sole electron acceptor. Geobacillus sp. strain S2E was isolated from anaerobic enrichments with $10 \mathrm{mM}$ acetate and $50 \mathrm{mM} \mathrm{Fe}$ (III) oxide following serial dilution to extinction five times. To obtain bacteria capable of using exogenous electron shuttles, electrode scrapings were inoculated into fresh water media with acetate $(10 \mathrm{mM})$ and AQDS $(10 \mathrm{mM})$. Isolation of Thermincola sp. strain JR and Coprothermobacter $s p$. strain COPO were performed by transferring scrapings of the anode biofilms into $9 \mathrm{ml}$ AQDS media under an anaerobic $\left(80: 20 \quad \mathrm{~N}_{2}: \mathrm{CO}_{2}\right)$ headspace. Colonies were isolated using the agar shaketube technique (Coates et al., 1996) with acetate serving as the electron donor and AQDS serving as the electron acceptor. Once pure cultures were identified, all isolated colonies were tested for ability to reduce poorly crystalline $\mathrm{Fe}(\mathrm{III})$ oxide. Strain JR and S2E were maintained on Fe(III) oxide, where COPO was maintained on AQDS.

\section{Analytical methods}

All experimental analyses were performed in triplicate to ensure reproducibility and the results are expressed as the mean of these determinations. The concentration of acetate and organic acids in the fuel cells were determined through high pressure liquid chromatography with a fast acid analysis column (Biorad, Hercules, CA, USA), operated with an eluent of $0.02 \mathrm{~N} \mathrm{H}_{2} \mathrm{SO}_{4}$ and UV detection @ $210 \mathrm{~nm}$.

\section{Nucleotide sequence accession numbers}

The sequence data of $16 \mathrm{~S}$ rRNA gene fragments have been submitted to the Genbank database with accession numbers EU638332-EU639378. 


\section{Results and discussion}

Electrochemical performance in a thermophilic MFC To evaluate the microbiology of anodic electron transfer under thermophilic conditions, we constructed and operated MFCs for over 100 days. Our experimental design included five reactors: two acetate-fed reactors, two unfed (no acetate) reactors and one acetate-fed open circuit control. Electrochemical performance was well replicated within the reactors; current harvesting was similar not only from replicates within this experiment but also to another independent experiment (data not shown). Polarization curves determined a maximum power of $37 \mathrm{~mW} \mathrm{~m}^{-2}$ and a $470 \mathrm{ohm}$ internal resistance. (Supplementary Figure 1). Current production was continuous; averaging $0.57 \mathrm{~mA}\left(100 \mathrm{~mA} \mathrm{~m}^{-2}\right.$; Figure 1) with a coulombic efficiency of $89 \%$ over more than 3 months of current harvesting for the two experimental reactors. As expected, the control reactors receiving no exogenous carbon amendment failed to produce significant current after 2 days (Figure 1).

PhyloChip analysis of community membership and dynamics

After 100 days of operation, the composition of the microbial community was assessed by excising biofilms from the electrodes. Nucleic acids (RNA and DNA) were extracted from the graphite matrices and the time zero inoculum. Total RNA, extracted from the current generating replicates and from the unfed reactor, was reverse transcribed to cDNA and PCR amplified along with genomic DNA from all reactors and the time zero inoculum. PCR amplification bias was minimized by using non-degenerate primers, 25 amplification cycles and pooling of PCR products from eight different annealing

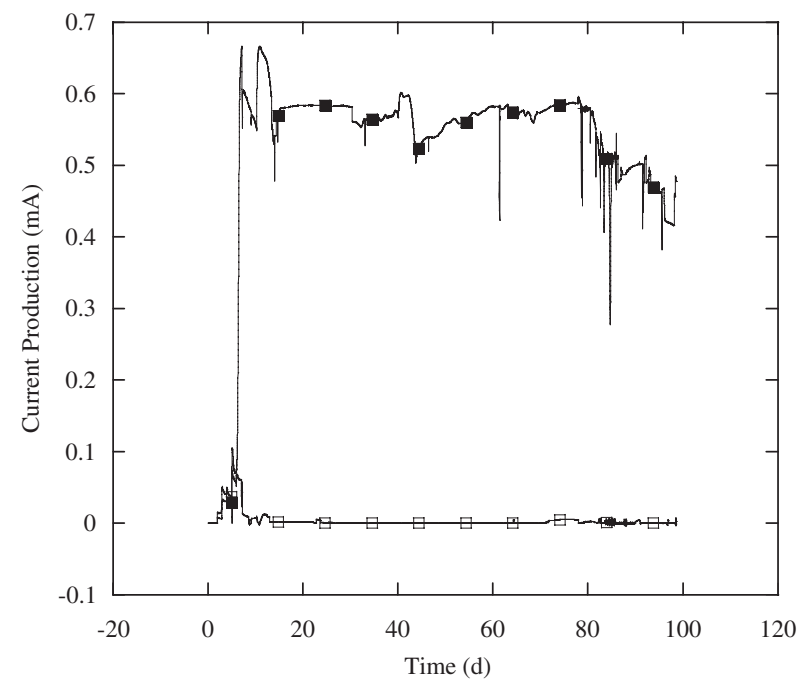

Figure 1 Electricity (current) produced by the anode bacterial community with ( $\mathbf{\square})$ and without acetate $(\square)$. temperatures (Brodie et al., 2007). Archaea were concluded to be an insignificant portion of the current-producing community as PCR amplification of extracted nucleic acids using specific primers failed to produce any products in the currentproducing reactors.

We performed a hierarchical cluster analysis on PhyloChip intensity data to compare the overall community structure within the current generating reactors and between the current and non-current reactors (Figure 2a). Three clusters were detected: no current controls, time zero inoculum and current generating community. The no current controls formed a separate cluster from the samples of the
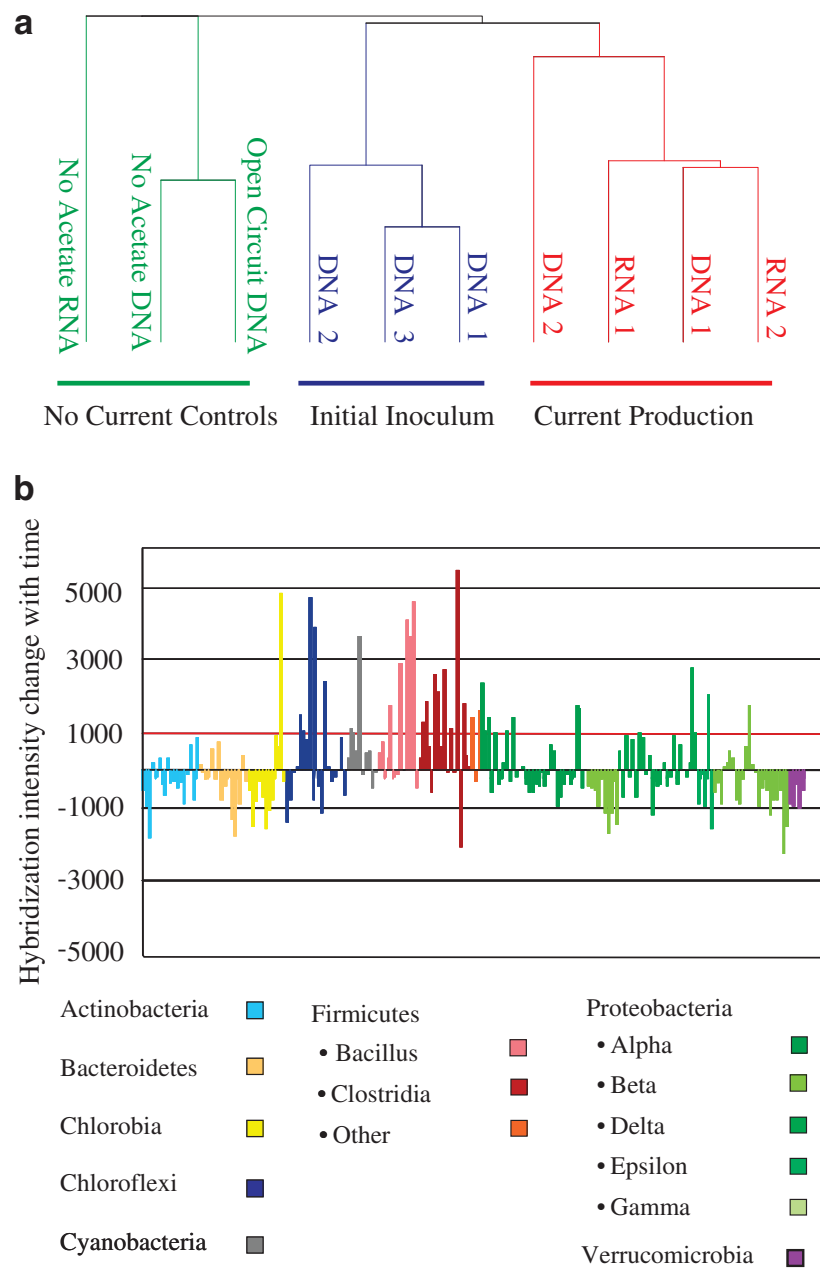

Figure 2 (a) Bacterial communities measured by PhyloChip hybridization intensity cluster into three distinct groups: initial inoculum, current-producing reactors and control reactors. (b) Changes in hybridization intensity between current-producing reactors and the initial inoculum identify bacterial taxa enriched in current-producing anodes. Bacteria are ordered alphabetically from left to right according to taxonomic affiliation (color coded). Bars above the zero line represent bacteria that increased in abundance relative to the initial inoculum; bars below represent those bacteria that decreased in abundance. An increase of 1000 hybridization units (red bar) is equivalent to a single log increase in relative abundance. 
initial inoculum and the final current-producing reactors, with the latter two arising from a common node indicating a population divergence resulting from the treatments. PhyloChip profiles with $16 \mathrm{~S}$ rDNA and 16S rRNA extracted from current-generating electrodes clustered together and represented similarly structured communities, whereas $16 \mathrm{~S}$ rDNA and 16S rRNA profiles from a control reactor failed to cluster (Figure 2a). Although some previous studies using anaerobic sludge bioreactors indicated a lack of phylogenetic reproducibility in functionally performing reactors (Fernandez et al., 2000; Collins et al., 2006), the 16S rDNA and 16S rRNA described communities in our two current-producing reactors were similar $(>89 \%)$ to each other. The high similarity of $16 \mathrm{~S}$ rDNA and $16 \mathrm{~S}$ rRNA communities from current-producing anodes is explained by the electrode enrichment process, with electrode colonization (16S rDNA) comprised of organisms that may grow by electron transfer $t$ o the anode or grow in association with active members of the active current-generating community (16S rRNA).

When analyzing the DNA PhyloChip arrays, we calculated and plotted the hybridization intensity score (HybScore) differences between each treatment and the initial inoculum to determine bacterial taxa enriched in current-producing reactors over time. Differences in HybScores are a direct measurement of changes in gene copy number with a 1000fold change in HybScore proportional to a single log change in relative abundance (Brodie et al., 2007). Despite the fact that overall bacterial richness decreased in all reactor samples with time, certain members of the bacterial electrode community increased in relative abundance from the initial inoculum (Figure 2b). Bacterial phyla increasing by at least one log in relative abundance in currentproducing reactors and decreasing in non-current controls comprised the dynamic subset (Supplementary Figure 2). Members of the Firmicutes (16 taxa, 38\%), Proteobacteria (11 taxa, 26\%), and Chloroflexi (five taxa, 12\%) were enriched only in current-producing reactors and constituted dominant members of the dynamic subgroup. Alternatively, in both of our non-current control reactors, taxa from the family Enterobacteriales of the gamma Proteobacteria increased significantly but did not increase in current-producing reactors. This indicates a metabolic role independent of power generation for members of the Enterobacteriales.

Our plan for tracking both the $16 \mathrm{~S}$ rDNA and $16 \mathrm{~S}$ rRNA was to link bacterial community structure with metabolic activity. This distinction is theoretically possible because the number of ribosomes in a cell is correlated to metabolic activity (Kerkhof and Kemp, 1999). To identify functionally active taxa, we performed a subtractive analysis using $16 \mathrm{~S}$ rRNA Hybscores from current-producing reactors and a non-current-producing control (no circuit treatment). Of the 14 taxa that increased significantly in PhyloChip RNA response (Supplementary Figure 3), 12 also increased significantly in PhyloChip DNA response outlined above. As a result, the $16 \mathrm{~S}$ rRNA analysis identified active populations within the dynamic $16 \mathrm{~S}$ rDNA subset. We conclude that analysis of both the 16S rRNA and 16S rDNA profiles is necessary for discerning the active subpopulations within the electrode bacterial community. Similar to the $16 \mathrm{~S}$ rDNA response, the $16 \mathrm{~S}$ rRNA response was dominated by the Firmicutes (seven taxa, 50\%) and Chloroflexi (three taxa, 21\%). Interestingly, even though the majority of known electricity-producing organisms are members of the gamma and delta subclasses of the Proteobacteria (Kim et al., 1999, 2002; Bond and Lovley, 2003; Rabaey et al., 2005), this phylum was not well represented in the $16 \mathrm{~S}$ rRNA subset. In fact, the relative abundance of Proteobacteria reduced from $26 \%$ in the DNA response to less than $7 \%$ of the RNA response (one taxon); indicating that dominantly detected Proteobacteria taxa may not have high levels of metabolic activity in the final currentproducing community in our fuel cells.

Together the 16S rDNA and rRNA PhyloChip results indicate that Firmicutes play a significant role in this MFC current-generating community. Although $16 \mathrm{~S}$ rDNA sequences belonging to the Firmicutes have previously been detected in the anode communities of active fuel cells (Lee et al., 2003; Kim et al., 2004, 2006; Rabaey et al., 2004, 2007; Aelterman et al., 2006; Hamid Rismani-Yazdi et al., 2007; Mathis et al., 2007), there are a limited number of publications where Firmicutes represent a dominant portion $(>50 \%)$ of the anode community composition (Rabaey et al., 2004, 2007; Aelterman et al., 2006; Hamid Rismani-Yazdi et al., 2007; Mathis et al., 2007). Previous studies of MFCs inoculated with Firmicutes isolates only produced current in the presence of an exogenous electron shuttle (Rabaey et al., 2005, 2007; Milliken and May, 2007; Pham et al., 2008) or as a byproduct of glucose fermentation (Park, 2001; Kim et al., 2005). Therefore, it was recently concluded that the presence of Gram-positive bacteria in an MFC is an outcome of ecological interaction with Gram-negative electricity-producing organisms rather than a functional interaction and electron transfer with the electrode surface (Rabaey et al., 2007).

\section{Dominant bacterial species identified}

To more specifically identify Firmicutes involved in current generation, we constructed 16S rDNA clone libraries from the initial inoculum and currentproducing reactors. Clone sequences (297) were produced from the current generating samples and were classified into 21 OTUs spanning six major bacterial lineages (Figure 3). Two Gram-positive phyla, Firmicutes (11 OTUs, 234 clones, $79 \%$ of clones) and Coprothermobacteria (two OTUs, 48 clones, $16 \%$ of clones), represented $95 \%$ of the 
clone sequences. Coprothermobacter species were previously identified by culture-independent molecular techniques in an MFC study with acetate as an electron donor and a thermophilic anaerobic digester inoculum (Jong et al., 2006). In that study,
Coprothermobacter sequences represented $15.1 \%$ of the community. However, the importance of monitoring bacterial community dynamics rather than assaying the final community was clearly illustrated by the fact that in our study Coprothermobacter

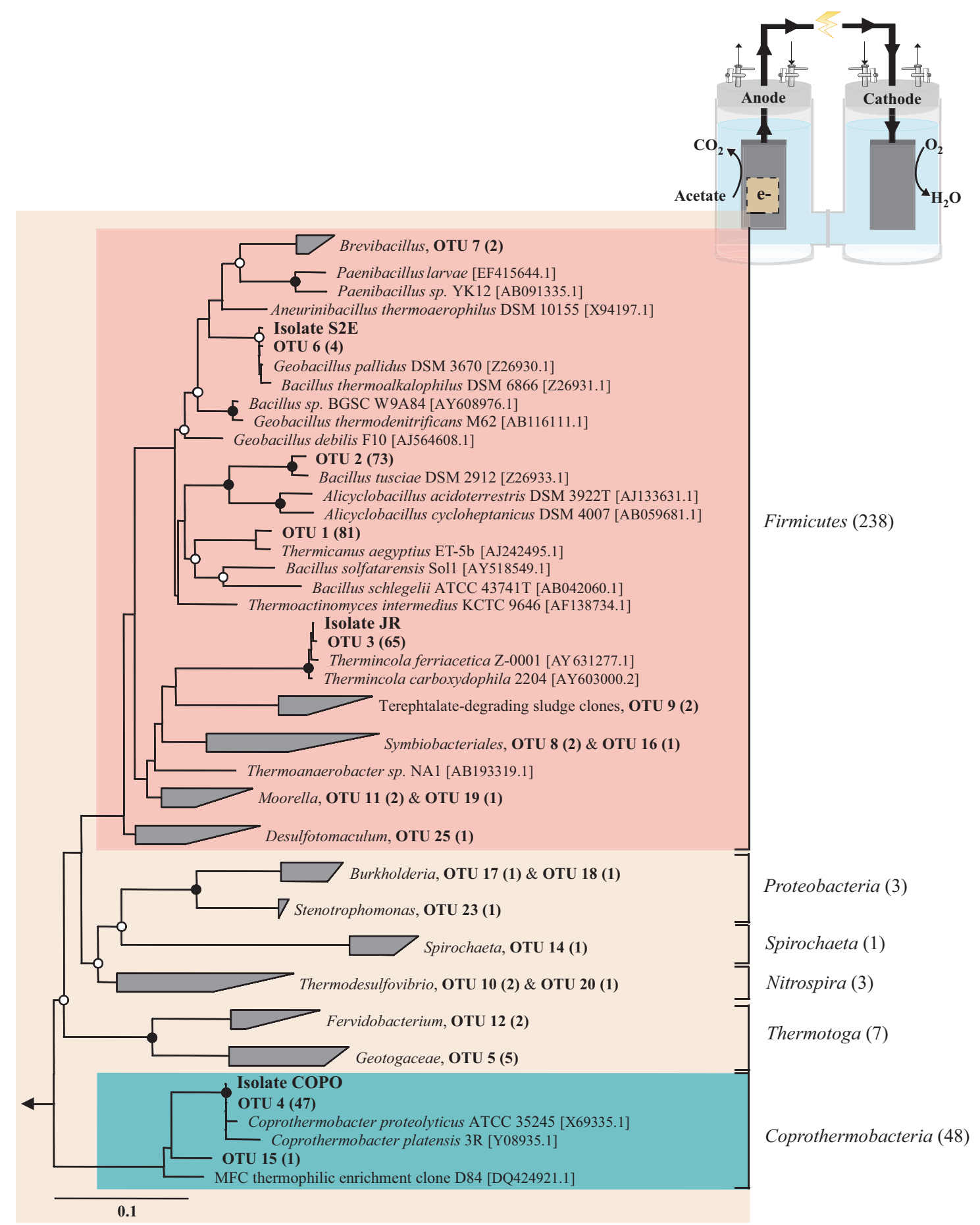

Figure 3 A maximum likelihood tree constructed from current-producing clone library sequences illustrates the phylogenetic position of $16 \mathrm{~S}$ rRNA gene clones. A total of 297 clones were sequenced representing 21 OTUs (bold) with the total number of sequences for each OTU in parentheses. Bootstrap values are denoted with circles with values greater than $70 \%(O)$ and greater than $90 \%(\bullet)$. Two dominant Phyla are denoted with colors; Firmicutes (pink box) and Coprothermobacteria (blue box). Sequences of the three electrode biofilm isolates are included on the tree (bold). The scale bar represents 0.1 inferred nucleotide changes per 100 nucleotides analyzed. 
Table 1 Total number and relative abundance of the five most frequent OTUs within clone libraries from the initial inoculum and electricity-producing anodes

\begin{tabular}{|c|c|c|c|c|c|}
\hline \multirow[t]{2}{*}{ OTU number } & \multirow[t]{2}{*}{ Genus } & \multicolumn{2}{|c|}{ Initial inoculum } & \multicolumn{2}{|c|}{ Current production } \\
\hline & & Total clones & $\%$ & Total clones & $\%$ \\
\hline OTU 1 & Thermicanus & 0 & 0 & 81 & 27 \\
\hline OTU 2 & Alicylobacillus & 0 & 0 & 73 & 25 \\
\hline OTU 3 & Thermincola & 2 & 0 & 65 & 22 \\
\hline OTU 4 & Coprothermobacter & 118 & 24 & 47 & 16 \\
\hline OTU 6 & Geobacillus & 0 & 0 & 4 & 2 \\
\hline
\end{tabular}

sequences decreased in relative abundance from the initial inoculum $(24 \%)$ to the final time point on current-producing anodes (16\%) (Table 1). This result implies that the presence of Coprothermobacter in thermophilic communities may be an artifact of their ability to use diverse electron donors independent of electron transfer to the anode rather than a selective enrichment in the MFC due to current production.

Within the Firmicutes, sequences belonging to the genera Thermicanus, Alicyclobacillus and Thermincola represented $27 \%, 25 \%$ and $22 \%$ of the total clones respectively. These three genera could not be detected in clone libraries from the initial inoculum (482 total clones) (Table 1), suggesting their enrichment is associated with current generation. Sequences related to Thermincola spp. have been detected in another electrode community (Mathis et al., 2007). In this study, MFCs were inoculated with marine sediment and operated at $60^{\circ} \mathrm{C}$ with acetate serving as the electron donor. Similar to our system, Firmicutes represented dominant members of the microbial community (64 of 80 clones; $80 \%$ ) with seven distinct phylotypes closely related $(88-99 \%$ similar) to Thermincola carboxydiphila (Mathis et al., 2007). This, combined with our results suggests that Thermincola species may have some unknown selective advantage over other bacteria in thermophilic MFC systems.

Here, we use two culture-independent methods in our analysis. Together they demonstrate the significant role of Firmicutes in the anode community expanding the recognized diversity of known electricity-producing organisms. Although the microarray approach detected over 35 times more bacterial types than the clone library sequencing, the PhyloChip data mirrored the clone library data. Owing to the limited number of clones that can feasibly be sampled from clone libraries, highly abundant species like the Firmicutes may mask the presence of less abundant but functionally significant species. For instance, members of the phylum Chloroflexi were enriched in both DNA and RNA dynamic subgroups in the PhyloChip analysis but were not identified in the clone library analysis (Brodie et al., 2006, 2007; DeSantis et al., 2007).
Bacterial isolates capable of anodic electron transfer In contrast to observations made with MFCs operated at mesophilic temperatures, our community analyses indicate a functional role for the Firmicute phylum in current production at $55^{\circ} \mathrm{C}$. Despite the large amount of data concerning the molecular identification of $16 \mathrm{~S}$ rRNA sequences in the environment, understanding the function of microbial communities is a major bottleneck in microbial ecology (Manefield et al., 2002). To conclusively link taxonomic identification with physiological function, we isolated bacteria capable of transferring electrons to the anode using media amended with amorphous hydrous ferric oxide (HFO) or 2,6-anthraquinone disulfonic acid (AQDS). Similar to an anode, HFO is an insoluble electron acceptor that should enrich for bacteria that can transfer electrons extracellularly without a requirement of exogenous electron shuttle (Gralnick and Newman, 2007). In contrast, AQDS should enrich for bacteria that can utilize an exogenous electron shuttle to indirectly reduce the anode and produce current.

Here, we report for the first time a culture-based approach to describe members of the thermophilic bacterial anode community. We isolated several bacteria representing three genera, Thermincola, Geobacillus and Coprothermobacter species. These genera represent three of the five most dominant members of the anode community and collectively represent $39 \%$ of the clone library sequence diversity (Figure 3). Both Firmicutes isolates, Thermincola sp. strain JR and Geobacillus sp. strain $\mathrm{S} 2 \mathrm{E}$, are of great interest given their significant enrichment from the initial inoculum (Table 1) and ability to reduce the solid phase electron acceptor (HFO). Although both isolates reduced solid phase iron coupled to acetate oxidation, only Thermincola sp. strain JR could generate current independent of an electron shuttle with acetate as an electron donor (Figure 4a). Strain JR generated an average of $0.42 \mathrm{~mA}$ (when normalized for background current) in two separate experiments with a coulombic efficiency of $91 \%$, similar to that observed for the original complex community $(0.57 \mathrm{~mA}, 89 \%)$. In comparison to similarly designed MFCs operated at $30^{\circ} \mathrm{C}$, Geobacter sulfurreducens produced a mean current of $0.251 \mathrm{~mA}$ $(n=3, \pm 0.04)$ from acetate oxidation (Bond and 

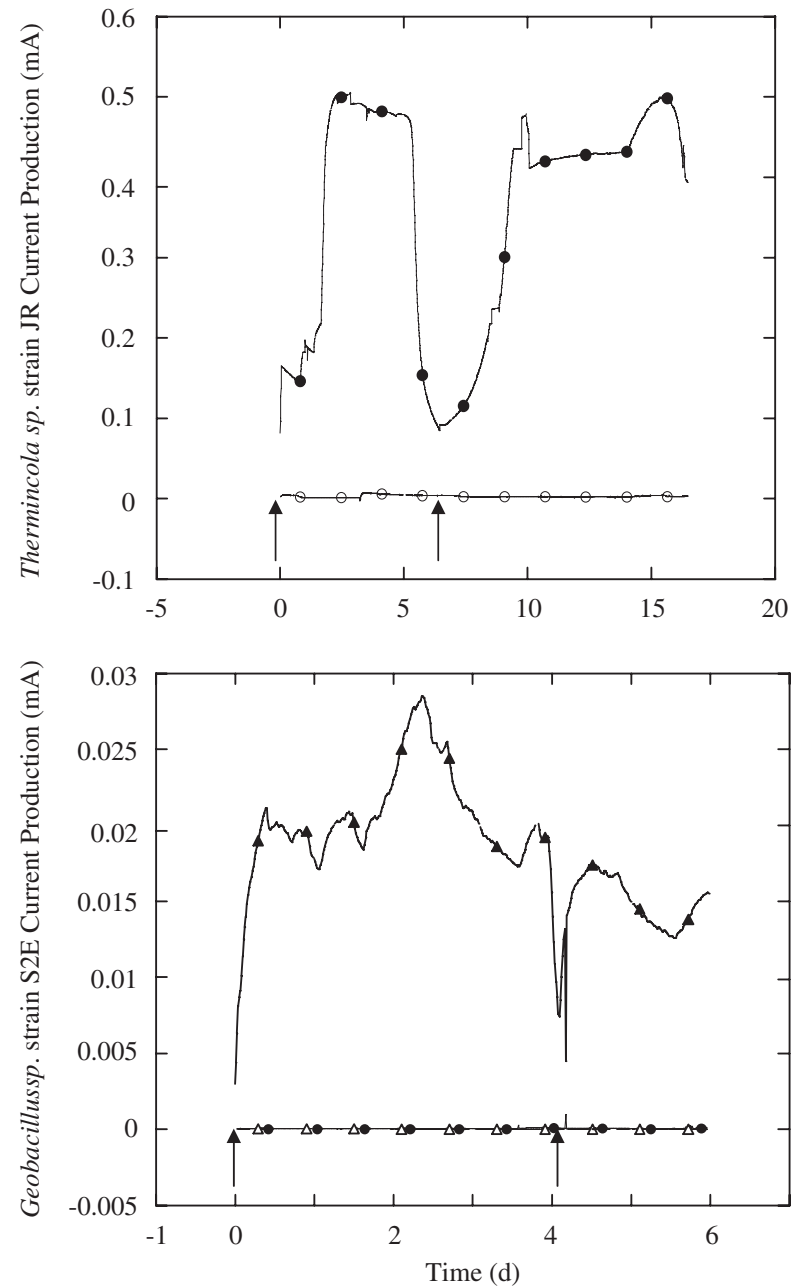

Figure 4 (a) Current produced by Thermincola sp. strain JR with $(\mathbf{O})$ and without $(O)$ acetate in the absence of an exogenous electron shuttle and (b) Geobacillus sp. strain S2E with electron shuttle (AQDS) and acetate $(\mathbf{\Delta})$ with electron shuttle without acetate $(\triangle)$ and without electron shuttle with acetate $(\bullet)$. Arrows indicate acetate amendment.

Lovley, 2003; Richter et al., 2007; JD Coates and KC Wrighton, unpublished data) and Shewanella onedensis strain MR-1 produced between 0.03-0.3 mA from lactate oxidation (Lanthier et al., 2008).

These studies show for the first time that Firmicutes can play a direct role in current production with acetate as the electron donor. Our results demonstrate that it is imperative that the role of Gram-positive bacteria in both anodic communities and the environment be redefined to include their ability to respire insoluble electron acceptors such as ferric iron or anodes. The data presented here demonstrates that the presence of Gram-positive bacteria in anodes is not an artifact of ecological interaction but a direct result of their ability to transfer electrons to the anode independent of exogenous electron shuttles. Future experiments exploring the mechanism of electron transfer will be an important next step in elucidating the role of Thermincola $s p$. strain JR within the anode biofilm.

In contrast to Thermincola sp. strain JR, Geobacillus sp. strain S2E could not produce current in the absence of an exogenous electron shuttle (AQDS) and only produced small amounts of current in its presence (0.03 mA) (Figure 4b). This data demonstrates that utilization of an insoluble electron acceptor does not directly translate to the ability of the bacterium to utilize an anode as an alternative electron acceptor (Kim et al., 2005). A similar discrepancy between HFO and anode reduction was recently observed by Richter et al. (2007) using the mesophilic Fe(III)-reducing organism Pelobacter carbinolicus. Reasons for this discrepancy demand further attention, and may be attributed to an unfavorable anode redox potential, or possibly the production of ligands by strain S2E similarly to the Fe(III)-reducing Acidobacteria Geothrix fermentans (Nevin and Lovley, 2002).

The results of our culture-based investigation demonstrate the novel role of the Firmicutes in electricity generation. Given the broader diversity of other organisms identified in culture-independent analyses, it is highly likely there are additional species that can transfer electrons directly to the electrode. Although not normalized to individual cell numbers, current production by strain JR alone accounts for approximately $70 \%$ of the amount produced by the entire community. This supports the notion that the increased current generation by the community may be due to the additional activity of other bacteria not yet identified in pure culture or by the synergistic electron transfer activities within the biofilm. Additionally, the importance of synergistic activity in electrochemically active biofilms has been demonstrated in mesophilic MFCs. Here, electrochemically inactive isolates exploited the redox shuttle produced by electrochemically active isolates (Rabaey et al., 2005, 2007; Pham et al., 2008). It is plausible that bacteria like Geobacillus spp. can function correspondingly in the thermophilic anode community identified here.

\section{Final conclusions}

Altogether, our findings demonstrate that thermophilic MFCs offer the opportunity to uncover hidden microbial diversity and function. We use both DNA- and RNA-based approaches as well as pure culture studies to link taxonomic identification of the community to a functional role in current production. Here, we show the insight gained through a combined application of cultivation and cultivation-independent methods to comprehensively characterize the structure and function of microbial communities.

Our results demonstrate that methods like the PhyloChip can uncover 35 times the diversity previously identified user lower resolution techniques like DGGE and clone library analysis. Complementary to the PhyloChip method, clone libraries identified the dominance of Firmicutes in our anode 
biofilms. Culture-based methods isolated dominant Firmicutes and confirm a previously unidentified electricity-producing role for members of this phylum. Independent anodic electron transfer by Firmicutes is consistent with physiological studies demonstrating that isolated members of this phylum can transfer electrons to solid phase electron acceptors, including iron and manganese, as part of a respiratory metabolism (Lovley et al., 2004; Weber et al., 2006; Zavarzina et al., 2007). Future physiological studies show promise toward unraveling the complexity of interactions in the anode biofilm as well as exploring mechanism of electron transfer to solid phase electron acceptors by Gram-positive bacteria.

\section{Acknowledgements}

Funding for this work was provided to JDC through the DOE Laboratory Directed Research and Development (LDRD) program. Part of this work was performed under the auspices of Lawrence Berkeley National Laboratory through contract DE-AC02-05CH11231 between Lawrence Berkeley National Laboratory and the US Department of Energy. We are indebted to Yvette Piceno and Julita Madejska for expertize with the PhyloChip and clone libraries, respectively. We thank Derek Lovley and his lab members Kelly Nevin, Sean Covalla and Jessica Johnson for technical guidance regarding microbial fuel cells. We appreciate the time David Coates spent on international phone calls sharing his knowledge of electrochemistry. We are also grateful to Cameron Thrash, Forest Kaser, Rebecca Daly and Kristen DeAngelis for informative discussions regarding microbial physiology, electrochemistry, phylogenetic structure and community assembly. Finally, we thank John Hake and Ryoko Kataoka of East Bay MUD for donating the thermophilic anaerobic digester sludge.

\section{References}

Aelterman P, Rabaey K, Pham HT, Boon N, Verstraete W. (2006). Continuous electricity generation at high voltages and currents using stacked microbial fuel cells. Environ Sci Technol 40: 3388-3394.

Bond DR, Holmes DE, Tender LM, Lovley DR. (2002). Electrode-reducing microorganisms that harvest energy from marine sediments. Science 295: 483-485.

Bond DR, Lovley DR. (2003). Electricity production by Geobacter sulfurreducens attached to electrodes. Appl Environ Microbiol 69: 1548-1555.

Bretschger O, Obraztsova A, Sturm CA, Chang IS, Gorby YA, Reed SB et al. (2007). Current production and metal oxide reduction by Shewanella oneidensis MR-1 wild type and mutants. Appl Enviro Microbiol 73: 7003-7012.

Brodie EL, DeSantis TZ, Joyner DC, Baek SM, Larsen JT, Andersen GL et al. (2006). Application of a highdensity oligonucleotide microarray approach to study bacterial population dynamics during uranium reduction and reoxidation. Appl Enviro Microbiol 72: 6288-6298.

Brodie EL, DeSantis TZ, Parker JPM, Zubietta IX, Piceno YM, Andersen GL. (2007). Urban aerosols harbor diverse and dynamic bacterial populations. PNAS 104: 299-304.

Chaudhuri SK, Lovley DR. (2003). Electricity generation by direct oxidation of glucose in mediatorless microbial fuel cells. Nat Biotech 21: 1229-1232.

Coates JD, Phillips EJ, Lonergan DJ, Jenter H, Lovley DR. (1996). Isolation of Geobacter species from diverse sedimentary environments. Appl Environ Microbiol 62: 1531-1536.

Collins G, Mahony T, O’Flaherty V. (2006). Stability and reproducibility of low-temperature anaerobic biological wastewater treatment. FEMS Microbiol Ecol 55: 449-458.

DeSantis T, Brodie E, Moberg J, Zubieta I, Piceno Y, Andersen G. (2007). High-density universal 16S rRNA microarray analysis reveals broader diversity than typical clone library when sampling the environment. Microb Ecol 53: 371-383.

DeSantis TZ, Hugenholtz P, Larsen N, Rojas M, Brodie EL, Keller K et al. (2006). Greengenes, a chimera-checked $16 \mathrm{~S}$ rRNA gene database and workbench compatible with ARB. Appl Environ Microbiol 72: 5069-5072.

Duineveld BM, Kowalchuk GA, Keijzer A, van Elsas JD, van Veen JA. (2001). Analysis of bacterial communities in the rhizosphere of chrysanthemum via denaturing gradient gel electrophoresis of PCR-amplified 16S rRNA as well as DNA fragments coding for 16S rRNA. Appl Enviro Microbiol 67: 172-178.

Fernandez AS, Hashsham SA, Dollhopf SL, Raskin L, Glagoleva O, Dazzo FB et al. (2000). Flexible community structure correlates with stable community function in methanogenic bioreactor communities perturbed by glucose. Appl Enviro Microbiol 66: 4058-4067.

Flanagan JL, Brodie EL, Weng L, Lynch SV, Garcia O, Brown $\mathrm{R}$ et al. (2007). Loss of bacterial diversity during antibiotic treatment of intubated patients colonized with Pseudomonas aeruginosa. J Bacteriol 45: 1954-1962.

Gorby YA, Yanina S, McLean JS, Rosso KM, Moyles D, Dohnalkova A et al. (2006). Electrically conductive bacterial nanowires produced by Shewanella oneidensis strain MR-1 and other microorganisms. PNAS 103: 11358-11363.

Gralnick JA, Newman DK. (2007). Extracellular respiration. Mol Microbiol 65: 1-11.

Hamid Rismani-Yazdi ADC, Burk AD, Mark M, Zhongtang Yu, Olli HT. (2007). Electricity generation from cellulose by rumen microorganisms in microbial fuel cells. Biotechnol Bioeng 97: 1398-1407.

Holmes DE, Nicoll JS, Bond DR, Lovley DR. (2004). Potential role of a novel psychrotolerant member of the family Geobacteraceae, Geopsychrobacter electrodiphilus gen. nov., sp. nov., in electricity production by a marine sediment fuel cell. Appl Environ Microbiol 70: 6023-6030.

Jong BC, Kim BH, Chang IS, Liew PWY, Choo YF, Kang GS. (2006). Enrichment, performance, and microbial diversity of a thermophilic mediatorless microbial fuel cell. Environ Sci Technol 40: 6449-6454.

Kerkhof L, Kemp P. (1999). Small ribosomal RNA content in marine Proteobacteria during nonsteady-state growth. FEMS Microbiol Ecol 30: 253-260.

Kim BH, Ikeda T, Park HS, Kim HJ, Hyun MS, Kano K et al. (1999). Electrochemical activity of an Fe(III)-reducing bacterium, Shewanella putrefaciens IR-1, in the 
presence of alternative electron acceptors. Biotechnol Tech 13: 475-478.

Kim BH, Park HS, Kim HJ, Kim GT, Chang IS, Lee J et al. (2004). Enrichment of microbial community generating electricity using a fuel-cell-type electrochemical cell. Appl Microbiol Biot 63: 672-681.

Kim GT, Hyun MS, Chang IS, Kim HJ, Park HS, Kim BH et al. (2005). Dissimilatory $\mathrm{Fe}(\mathrm{III})$ reduction by an electrochemically active lactic acid bacterium phylogenetically related to Enterococcus gallinarum isolated from submerged soil. J Appl Microbiol 99: 978-987.

Kim GT, Webster G, Wimpenny JWT, Kim BH, Kim HJ, Weightman AJ. (2006). Bacterial community structure, compartmentalization and activity in a microbial fuel cell. J Appl Microbiol 101: 698-710.

Kim HJ, Park HS, Hyun MS, Chang IS, Kim M, Kim BH. (2002). A mediator-less microbial fuel cell using a metal reducing bacterium, Shewanella putrefaciens. Enzyme Microb Tech 30: 145-152.

Lanthier M, Gregory KB, Lovley DR. (2008). Growth with high planktonic biomass in Shewanella oneidensis fuel cells. FEMS Microbiol Lett 278: 29-35.

Lee J, Phung NT, Chang IS, Kim BH, Sung HC. (2003). Use of acetate for enrichment of electrochemically active microorganisms and their 16S rDNA analyses. FEMS Microbiol Lett 223: 185-191.

Lovley DR. (2000). Environmental Microbe-Metal Interactions, 1st edn. ASM Press: Washington, DC.

Lovley DR. (2006). Bug juice: harvesting electricity with microorganisms. Nat Rev Micro 4: 497-508.

Lovley DR, Holmes DE, Nevin K. (2004). Dissimilatory Fe(III) and Mn(IV) reduction. Adv Microb Physiol 49: 219-286.

Lovley DR, Philips EJ. (1986). Organic matter mineralization with the reduction of ferric iron in anaerobic sediments. Appl Environ Microbiol 51: 683-689.

Lueders T, Wagner B, Claus P, Friedrich MW. (2004). Stable isotope probing of rRNA and DNA reveals a dynamic methylotroph community and trophic interactions with fungi and protozoa in oxic rice field soil. FEMS Microbiol Lett 6: 60-72.

Manefield M, Whiteley AS, Griffiths RI, Bailey MJ. (2002). RNA stable isotope probing, a novel means of linking microbial community function to phylogeny. Appl Environ Microbiol 68: 5367-5373.

Mathis BJ, Marshall CW, Milliken C, Makkar RS, Creager SE, May HD. (2007). Electricity generation by thermophilic microorganisms from marine sediment. Appl Microb Cell Physio.

Milliken CE, May HD. (2007). Sustained generation of electricity by the spore-forming, Gram-positive,
Desulfitobacterium hafniense strain DCB2. Appl Microbiol Biotech 73: 1180-1189.

Nevin KP, Lovley DR. (2002). Mechanisms for accessing insoluble Fe(III) oxide during dissimilatory Fe(III) reduction by Geothrix fermentans. Appl Environ Microbiol 68: 2294-2299.

Park HS. (2001). A novel electrochemically active and Fe(III)-reducing bacterium phylogenetically related to Clostridium butyricum isolated from a microbial fuel cell. Anaerobe 7: 297-306.

Pham T, Boon N, Aelterman P, Clauwaert P, De Schamphelaire L, Vanhaecke L et al. (2008). Metabolites produced by Pseudomonas sp. enable a Gram-positive bacterium to achieve extracellular electron transfer. Appl Microbiol Biotech 77: 1119-1129.

Rabaey K, Boon N, Hofte M, Verstraete W. (2005). Microbial phenazine production enhances electron transfer in biofuel cells. Environ Sci Technol 39: 3401-3408.

Rabaey K, Boon N, Siciliano SD, Verhaege M, Verstraete W. (2004). Biofuel cells select for microbial consortia that self-mediate electron transfer. Appl Enviro Microbiol 70: 5373-5382.

Rabaey K, Rodriguez J, Blackall LL, Keller J, Gross P, Batstone D et al. (2007). Microbial ecology meets electrochemistry: electricity-driven and driving communities. ISME J 1: 9-18.

Reguera G, McCarthy KD, Mehta T, Nicoll JS, Tuominen MT, Lovley DR. (2005). Extracellular electron transfer via microbial nanowires. Nature 435: 1098-1101.

Richter H, Lanthier M, Nevin KP, Lovley DR. (2007). Lack of electricity production by Pelobacter carbinolicus indicates that the capacity for Fe(III) oxide reduction does not necessarily confer electron transfer ability to fuel cell anodes. Appl Environ Microbiol 73: 5347-5353.

Sekiguchi Y, Kamagata Y, Syutsubo K, Ohashi A, Harada H, Nakamura K. (1998). Phylogenetic diversity of mesophilic and thermophilic granular sludges determined by $16 \mathrm{~S}$ rRNA gene analysis. Microbiology 144: 2655-2665.

Thrash JC, VanTrump JI, Weber KA, Miller E, Achenbach LA, Coates JD. (2007). Electrochemical stimulation of microbial perchlorate reduction. Environ Sci Technol 41: 1740-1746.

Weber KA, Achenbach LA, Coates JD. (2006). Microorganisms pumping iron: anaerobic microbial iron oxidation and reduction. Nat Rev Micro 4: 752-764.

Zavarzina D, Sokolova T, Tourova T, Chernyh N, Kostrikina N, Bonch-Osmolovskaya E. (2007). Thermincola ferriacetica sp. nov., a new anaerobic, thermophilic, facultatively chemolithoautotrophic bacterium capable of dissimilatory Fe(III) reduction. Extremophiles 11: $1-7$.

Supplementary Information accompanies the paper on The ISME Journal website (http://www.nature.com/ismej) 